\title{
El nervio auriculotemporal. Bases neuroanatómicas del Síndrome de Frey
}

\author{
G. Decuadro-Sáenz; G. Castro; N. Sorrenti; I. Doassans; S. Deleon; F. Salle; A. Saibene; A. Santamaría; A. Pérez- \\ Brignani* y V.R. Soria-Vargas
}

Departamento de Anatomía. Facultad de Medicina. Universidad de la República. Montevideo. Uruguay. *Clínica de Dolor Facial. Departamento de Fisiatría Dr. Fernando Acle. Hospital Policial. Ministerio del Interior. Montevideo. Uruguay.

\section{Resumen}

El propósito de este estudio fue precisar las relaciones del nervio auriculotemporal en las regiones infratemporal y parotídea. Se realizó la microdisección de 32 regiones infratemporales y parotídeas de cadáveres humanos formolados. Los patrones de origen fueron: , raíz única: $15.4 \%$, dos raíces: $73.1 \%$ y tres raíces: $11.5 \%$. En todos los casos la raíz lateral era la de mayor diámetro (2-2,5 mm). En los casos con dos raíces estas constituían un ojal nervioso a la arteria meníngea media en el $89.4 \%$ de los casos. La raíz medial se originaba en todos los casos del borde posterior del segmento inicial del nervio alveolar inferior. La distancia media entre la raíz lateral del nervio auriculotemporal y la cápsula de la articulación temporomandibular fue de $1.5 \mathrm{~mm}$, con patrón asimétrico, derecha: $2 \mathrm{~mm}$ (rango: 0-7 mm; DE $1.90 \mathrm{~mm}$ ); izquierda: $1.25 \mathrm{~mm}$ (rango: 0-3 mm; DE 0.88 mm). En 10 casos (31.3\%) la raíz lateral se encontraba en contacto directo con la cápsula articular, izquierda (46.1\%) y a derecha (21.1\%). La distancia media en la cara posterior de la articulación temporomandibular, desde la cara superior del disco articular al tronco del nervio auriculotemporal fue de 10,5 mm (DE: $4.06 \mathrm{~mm}$ ), con patrón asimétrico, derecha: $9 \mathrm{~mm}$ (rango: 5,0-18,0 $\mathrm{mm}$; DE 3,70 mm), izquierda: $11.0 \mathrm{~mm}$ (rango: 3.0-20.0 $\mathrm{mm}$; DE $4.41 \mathrm{~mm})$. Se discute el rol de estos hallazgos en la etiología del síndrome de Frey.

PALABRAS CLAVE: Nervus auriculo temporalis. Articulatio temporomandibularis. Glandula parotis. Síndrome de Frey. Síndrome auriculotemporal.

Auriculo temporal nerve. Neuroanatomic bases of the Frey' Syndrome

\section{Summary}

The aim of this study was to precise the relations-

Recibido: 14-07-07. Aceptado: 2-10-07 hips of the auriculotemporal nerve in the infratemporal and parotid regions. We realized micro-dissections of thirty-two infratemporal and parotid regions of human cadaver's formol-fixed. The pattern of origin was: one root: $15.4 \%$, two roots: $73.1 \%$, three roots: $11.5 \%$. In all cases, the lateral root present the major diameter $(2-2,5 \mathrm{~mm})$. In those cases with two roots, these formed a neural loop round the middle meningeal artery in the $89.4 \%$ of the cases. The origin of the medial root was the posterior border of the inferior alveolar nerve. The median distance between the lateral root and the temporomandibular joint capsule was $1.5 \mathrm{~mm}$, with asymmetric pattern: right: $2 \mathrm{~mm}$ (rank: 0-7 mm; ED:

Abreviaturas. AMI: Arteria maxillaris (Arteria maxilar). Amm: Arteria meníngea media. Ata: Arteria tympánica anterior. ATM: Articulatio temporomandibularis (Articulación temporomandibular). ATS: Arteria temporalis superficialis (Arteria temporal superficial). CM: Condylus mandibularis (Cóndilo mandibular). DA: Discus articularis. (Disco articular). FI-MPL: Fasciculus inferioris. Mm pterygoidei. (Fascículo inferior del músculo pterigoideo lateral). FS-MPL: Fasciculus superioris. Mm pterygoidei. (Fascículo superior del músculo pterigoideo lateral). Go: Ganglion oticum (Ganglio otico). GP: Glandula parotis. (Glándula parotida). MPL: Musculus pterygoidei lateralis (Músculo pterigoideo lateral). NAI: Nervus alveolaris inferior (Nervio alveolar inferior). NAT: Nervus auriculo temporalis (Nervio auriculotemporal). NB: Nervus buccalis (Nervio bucal). NL: Nervus lingualis. (Nervio lingual). NM: Nervus massetericus (Nervio maseterino). NPm: Nervus pterygoideus medialis (Nervio del pterigoideo medial). NTPa: Nervus temporalis profundus (ramus anterior) (Nervio temporal profundo anterior). NTPm: Nervus temporalis profundus (ramus medius) (Nervio temporal profundo medio). NTPp: Nervus temporalis profundus (ramus posterior) (Nervio temporal profundo posterior). RextNAT: Radix externa Nervus auriculo temporalis (Raiz externa del NAT). RimNAT: Radix intermedius Nervus auriculo temporalis (Raiz intermedia NAT). RintNAT: Radix interna Nervus auriculo temporalis (Raiz interna del NAT). SF: Síndrome de Frey. V2: Nervus maxillaris (Nervio maxilar). V3: Nervus mandibularis. (Nervio mandibular). VII: Nervus facialis. (Nervio facial). VMI: Vena maxillaris (Vena maxilar) 
1.90mm); left: $1.25 \mathrm{~mm}$ (rank: 0-3 mm; ED: $0.88 \mathrm{~mm}$ ). In 10 cases $(31.3 \%)$ the lateral root was in direct contact with the articular capsule, with asymmetric pattern: left: $46.1 \%$; right: $21 \%$. The median distance between the superior aspect of the articular disk and the trunk of the auriculotemporal nerve in the posterior aspect of the temporomandibular joint was $10,5 \mathrm{~mm}$ (ED: 4.06 $\mathrm{mm}$ ), with asymmetric pattern: right: $9 \mathrm{~mm}$ (rank: 5$18.0 \mathrm{~mm}$; ED: $3.70 \mathrm{~mm}$ ); left: $11.0 \mathrm{~mm}$ (rank: 3.0-20 $\mathrm{mm}$; ED: $4.41 \mathrm{~mm}$ ). We discussed the rol of this findings in the etiology of the Frey's syndrome.

KEY WORDS: Auriculotemporal nerve. Temporomandibular joint. Parotid gland. Frey's syndrome. Gustatory flushing.

\section{Introducción}

El nervio mandibular (Nervus mandibularis, V3) es la tercera rama terminal del nervio trigémino ( $\mathrm{V}$ par craneal). Única rama terminal con componente motor, representa la principal fuente de inervación motora de los músculos masticatorios, con excepción de los asociados al hueso hioides. A unos 4-5 mm por debajo del foramen oval V3 se distribuye en forma terminal en un patrón de distribución variable según la conceptualización de diferentes anatomistas clásicos: 1-patrón terminal en 7 ramos terminales agrupados en: lateral, posteriores, inferiores y mediales ${ }^{15,42,54,66}, 2$-patrón arborescente en 6 ramos colaterales y 2 ramos terminales, los nervios alveolar inferior (NAI) y lingual (NL) ${ }^{63}$; 3-patrón torcular en el cual, luego de otorgar un ramo colateral recurrente meníngeo, se divide en dos cortos troncos nerviosos: tronco anterior, origen de los ramos laterales: nervio bucal (Nervus buccalis, NB;) el cual da origen al nervio temporal profundo anterior (Nervus temporalis profundus (ramus anterior), NTPa), nervio temporal profundo medio (Nervus temporalis profundus (ramus medius), NTPm) y nervio maseterino (Nervus massetericus, NM;), el cual da origen al nervio temporal profundo posterior (Nervus temporalis profundus (ramus posterior), NTPp); y tronco posterior que da origen a un ramo interno, el nervio del pterigoideo medial (Nervus pterygoideus medialis, NPm), a un ramo posterior el nervio auriculotemporal (Nervus auriculo temporalis, NAT), y dos ramos inferiores, el nervio lingual (Nervus lingualis, NL) y el nervio alveolar inferior (Nervus alveolaris inferior, NAI) $)^{7,8,30,47,50,61}$.

El nervio auriculotemporal (Nervus auriculo temporalis; NAT) es un nervio constituido por fibras nerviosas sensitivas y vegetativas. Proporciona la inervación sensitiva, (propioceptiva y nociceptiva), de la articulación temporomandibular (Articulatio temporomandibularis, ATM), la mucosa del oído medio, membrana timpánica, ligamentos del hueso martillo, superficie anterior de la coclea, conducto auditivo externo, y de la piel de las regiones temporal pterional y pabellón auricu- lar. Su rol vegetativo comprende la inervación parasimpática y simpática de la ATM, la inervación parasimpática de la glándula parótida (Glandula parotis, GP), la inervación simpática de las arterias meníngea media (Arteria meníngea media, Amm), timpánica anterior (Arteria tympánica anterior, Ata) y temporal superficial (Arteria temporalis superficialis, ATS), y la inervación simpática y parasimpática de las arterias y anexos de la piel de las regiones temporal, pterional y pabellón auricular $5,7,8,15,17,30,37,38,42,47,50,54,59,61,63,66$. El conocimiento de la anatomía topográfica del NAT posee una nueva vigencia en el marco de las disfunciones temporo-mandibulares, los traumatismos maxilofaciales, la cirugía de la base de cráneo, ATM y GP, así cmo en la interpretación de los síndromes neurológicos periféricos consecutivos a su lesión ${ }^{17}$. Las investigaciones existentes sobre este nervio se centran en su patrón de origen y sus relaciones con el cuello y cóndilo mandibular (Condylus mandibularis, CM) 16,17,25,28,32,40,49,55,56,64. Estos estudios son difícilmente comparables en sus hallazgos ya que como metodología utilizan diferentes técnicas de abordaje al NAT, como ser el abordaje lateral trans-zigomático, el abordaje superior (de Pinto) y el abordaje combinado lateral y superior. Como crítica a los mismos debemos destacar que el abordaje lateral altera en gran medida la anatomía topográfica normal de la región y el abordaje de Pinto no permite más que una visión muy limitada de la región infratemporal medial al músculo pterigoideo lateral. Las relaciones mediales están implicadas en la génesis de diversos síntomas óticos presentes en el curso de las disfunciones internas de la ATM (sindrome otomandibular, síndrome de Costen $)^{6,16,17,32,49,56}$, las relaciones posteriores y laterales son de interés en la elaboración y ejecución del bloqueo anestésico del NAT ${ }^{64}$, en el abordaje quirúrgico tranzygomático de la base de cráne ${ }^{67}$, en el abordaje quirúrgico de la ATM y de la glándula parótida ${ }^{1}$. La inervación vegetativa de la GP ha sido objeto de numerosas investigaciones en el campo de la fisiología e histología en animales y en el ser humano $2,9,11,19,20,22,23,26,29,34,44,46$, Aigrot $^{2}$ fue el primero en demostrar el rol vegetativo del nervio auriculotemporal en la inervación secretora de la GP, lo que fue confirmado posteriormente por Emmelin ${ }^{22}$. El estudio de las alteraciones sufridas por la GP ante la sección del NAT en la región infratemporal del gato, permitió evidenciar la existencia de inervación noadrenérgica no-colinérgica de la misma transportada también por este nervio ${ }^{20}$. Sin embargo, no existen estudios que describan la anatomía topográfica y descriptiva del NAT en relación con su función secretoria en la GP.

El síndrome de Frey (SF: síndrome de Frey-Baillarger, gustatory flushing, gustatory sweating, sindrome auriculotemporal) es la expresión clínica de una neuropatía vegetativa que tiene como causa la lesión mecánica o irritativa de las fibras vegetativas del NAT en su trayecto in fratemporal ${ }^{10,13,18,27,36,39,43,48,53,62}$. Fue descrito por primera vez como síndrome en 1923 por Lucja Frey, pero la observación de estos signos y síntomas se remonta al año 1740 por Kas- 
tremsky ${ }^{18}$. Se caracteriza por la aparición de hiperestesia, eritema, rubor, calor y sudoración en el territorio de piel inervado por el NAT, asociado a hipersalivación, luego de la aplicación de estímulos gustativos sobre el dorso de la lengua, durante el acto de la masticación o la succión, así como al imaginar un sabor, olor o alimento y en respuesta al arco reflejo salival. Se ha descrito en niños con antecedentes de parto distócico con utilización de fórceps ${ }^{36,39,43,48}$, $\mathrm{y}$ en adultos con fracturas condilares ${ }^{13,62}$, o posteriormente al abordaje quirúrgico de la $\mathrm{GP}^{1,13,53}$, de la $\mathrm{ATM}^{13,18,62}$, de la glándula submandibular ${ }^{13} \mathrm{y}$ de los ganglios linfáticos cervicales $^{13}$, así como en pacientes con diabetes mellitus ${ }^{26}$.

El presente estudio tiene como objetivo precisar las relaciones del NAT en las regiones infratemporal y parotídea, con el objetivo de definir las bases neuroanatómicas del SF que permitan prevenir esta neuropatía vegetativa en el curso de las cirugías de la base de cráneo, ATM y GP.

\section{Material y métodos}

Se disecaron 32 regiones infratemporales, 19 derechas y 13 izquierdas, procedentes de cadáveres adultos formolados de ambos sexos, de edades entre 40-90 años, sin antecedentes de patología traumática, mal formativa u otorrinolaringológica. Las mismas fueron procesadas bajo las normas de inyección y conservación cadavérica del Departamento de Anatomía de la Facultad de Medicina de la Universidad de la República Oriental del Uruguay. Se acondicionó el cadáver fresco, se abordaron los vasos femorales comunes a nivel del triángulo de Scarpa, realizándose su descubierta y la colocación de dos gruesas cánulas, procediendo al lavado del sistema arteriovenoso con una solución salina de ácido acético al 15\% mediante un sistema de bomba de impulsión favorecido por el efecto de la gravedad. Previo retiro de la cánula venosa y ligadura de la vena, se inyectaron 7-9 litros de solución fijadora, por vía arterial, primero en dirección centrífuga y luego en dirección centrípeta, mediante un sistema de irrigador o bomba de impulsión bajo la fuerza de gravedad. Se utilizó para esto la Solución Montevideo, solución de fijación y conservación creada y aplicada en forma sistemática por el Departamento de Anatomía de la Facultad de Medicina de la Universidad de la República Oriental del Uruguay. La inyección se considero completa cuando se evidencio cierto aumento de volumen en los miembros con signos similares a los de un edema gravitacional. Se conservaron los cadáveres mediante sumersión en una tina con el mismo líquido fijador, durante un plazo variable no mayor a dos semanas. Se seccionó y separó la cabeza del cuello mediante un corte transversal en la articulación entre la cuarta y tercera vértebra cervical. Previo decalotamiento, se extrajeron los hemisferios cerebrales y menínges del piso supratentorial de la cavidad craneal, con conservación en su posición original del tronco encefálico y raíces de los nervios craneales. Seguidamente se procedió al corte sagital de las cabezas. En cada hemicabeza, se realizó un doble abordaje por vía medial y superior de la región infra-temporal, entendiendo por tal aquella localizada por debajo de la fosa temporal del piso medio de la base de cráneo, por detrás de la cara posterior del maxilar superior, por delante y debajo de la cara exocraneana del peñasco del hueso temporal, medial a la rama montante de la mandíbula y la ATM y lateral de la rinofaringe. En un primer paso el abordaje medial se realizó mediante la disección y fresado del cuerpo y ala mayor del hueso esfenoides de medial a lateral hasta exponer el contenido del foramen oval y el foramen spinosum, se desinsertó el músculo pterigoideo medial de la base de la apófisis pterigoides, y se seccionó el ligamento esfenomandibular, reclinando la aponeurosis inter-pterigoidea de atrás hacia delante y de abajo a arriba, lo que permitió acceder a la celda externa de la fosa infratemporal. En un segundo paso el abordaje superior se realizó mediante la apertura del techo de la fosa infra-temporal en base al fresado y disección del ala mayor del esfenoides y escama del hueso temporal a partir del borde lateral del foramen oval y del foramen spinosum continuando en sentido lateral, procediendo a la extracción del ala mayor del esfenoides, la eminencia articular, y la escama del hueso temporal. Para estas microdisecciones se utilizó material de ampliación visual dado por 2 Lupas de Micro-disección (Olympus), con luz, y microscopio quirúrgico (Olympus $O M E$ ) con lentes Ernst Leitz Gm5H Welzlar con ampliación de $12.5 x$ y $15 x$. Con el nervio expuesto se registró: 1) patrón de distribución terminal de $\mathrm{V} 3 ; 2$ ) el número de raíces de origen del NAT; 3) relación de las raíces del nervio con la Amm; 4) distancia transversal desde la cara medial de la cápsula articular de la ATM a la cara externa de la raíz lateral del NAT (Rext NAT); 5) distancia vertical desde el polo medial del disco articular a la cara superior de la raíz lateral del NAT (RextNAT); 6) relaciones del NAT a nivel del ojal retrocondileo de Juvara; 7) relación del tronco del NAT con la cara posterior de la ATM; 8) distancia desde la cara superior del disco articular a la cara superior del tronco del NAT en la cara posterior de la ATM; 9) relaciones del tronco del NAT con la ATS y el tronco de VII en la región parotídea; y 10) ramos colaterales del NAT. Las medidas fueron realizadas con cartabón milimetrado con resolución de $1 / 20 \mathrm{~mm}$ y $1 / 128$ pulgadas.

\section{Resultados}

\section{Patrón de distribución terminal de V3}

Se registró el patrón de distribución terminal de V3 en 20 casos.

En 14 casos (70\%) V3 se dividía en 7 ramos terminales, sin identificarse un tronco anterior y un tronco posterior. En 6 casos $(30 \%) \mathrm{V} 3$ se bifurcaba en dos troncos terminales, antero-externo y postero-interno. El tronco antero-externo era menor o igual a $5 \mathrm{~mm}$ de longitud y daba origen en todos estos casos a los ramos laterales de V3. Estos patro- 

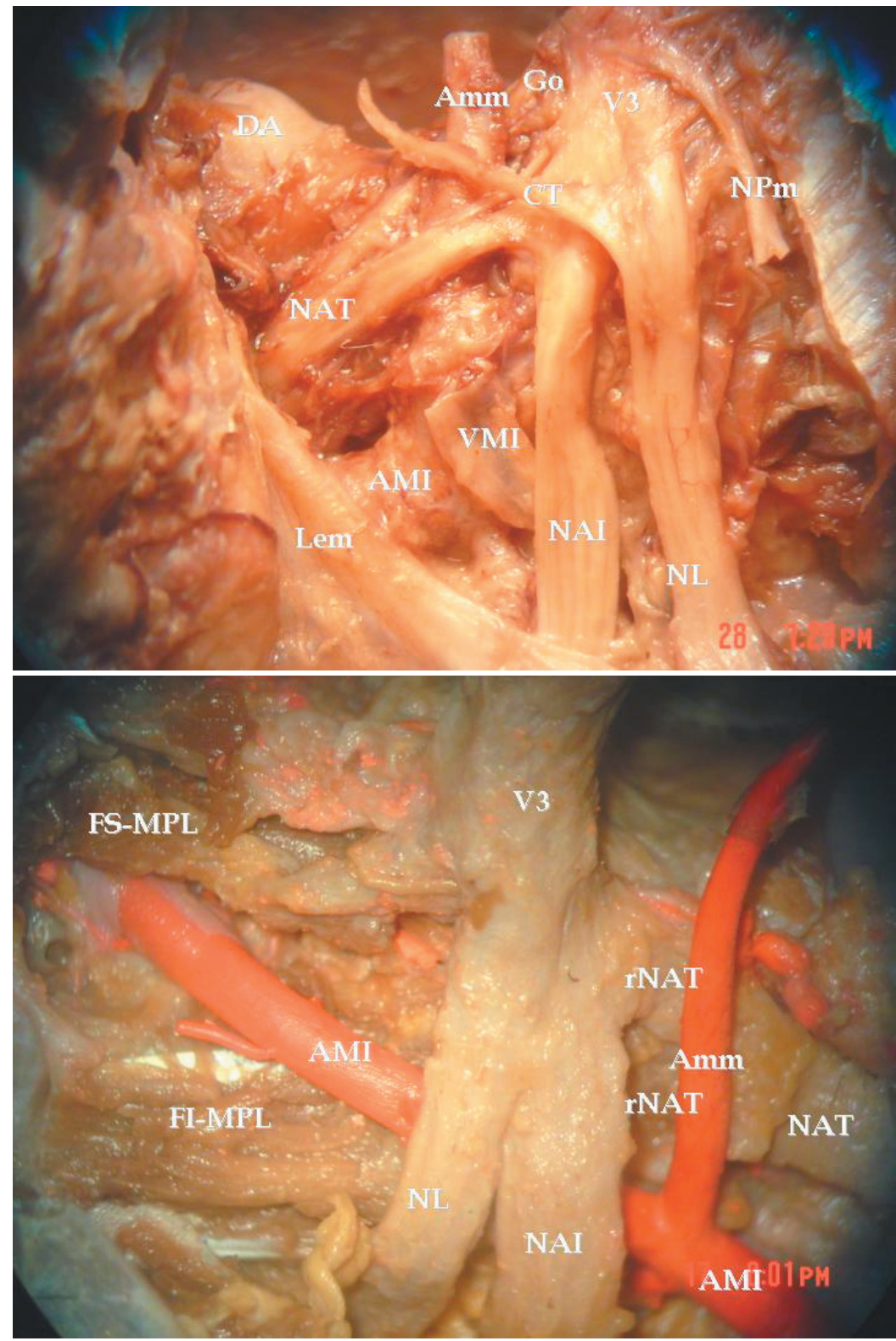

Figura 1. Microdisección de región infratemporal izquierda (Vista medial. Microscopio quirúrgico Olympus. Ampliación x15). Se observa un NAT con dos raíces de origen y el ojal que forman a la Amm Se observa un ramo anastomótico con el ganglio ótico. AMI: Arteria maxillaris. Amm: Arteria meningea media. CT: Chorda tympani. DA: Discus articularis. Go: Ganglion oticum. Lem: Ligamentus sphenomandibularis. NAI: Nervus alveolaris inferior. NAT: Nervus auriculo temporalis. NL: Nervus lingualis. RextNAT: Radix externa NAT. RintNAT: Radix interna NAT. V3: Nervus mandibularis. VMI: Vena maxillaris.

Figura 2. Microdisección de región infratemporal derecha. (Vista medial. Microscopio quirúrgico Olympus. Ampliación x15). Se observa un NAT con una única raíz de origen lateral a la Amm. AMI: Arteria maxillaris. Amm: Arteria meningea media. FI-MPL: Fasciculus inferioris. Mm pterygoidei. FS-MPL: Fasciculus superioris. Mm pterygoidei. NAI: Nervus alveolaris inferior. NAT: Nervus auriculo temporalis. NL: Nervus lingualis. rNAT: Radix NAT. V3: Nervus mandibularis. nes de terminación no evidenciaron patrones de asimetría significativas izquierda-derecha.

\section{Raíces del NATy sus relaciones con la Amm}

La anatomía descriptiva de las raíces del NAT se registró en 26 casos, debiéndose excluir 6 casos por problemas técnicos de disección; las relaciones con la Amm se estudiaron en 25 casos, debiéndose excluir 7 casos por problemas técnicos de disección. En 19 casos (73.1\%) el origen del NAT estaba representado por dos raíces, una lateral y superior, y otra medial e inferior, siendo esto más fracuente a izquierda $(77.8 \%)$ que a derecha $(70.6 \%)$. En todos estos casos la RextNAT era la de mayor diámetro $(2,0-2,5 \mathrm{~mm})$, se originaba del V3 como un ramo colateral posterior, y se dirigía de adelante a atrás pasando entre la Amm, medialmente, y el músculo pterigoideo lateral (Musculus pterygoidei lateralis, MPL), lateralmente. La raíz medial del NAT (RintNAT) se originaba en todos los casos del borde posterior del segmento inicial del NAI, y se dirigía de adelante a atrás pasando entre la AMM, lateralmente, y la aponeurosis interpterigoidea, medialmente la cual tapizaba la cara externa del músculo pterigoideo medial.

Con respecto al trayecto y relaciones de estas dos raíces en 17 casos ( $89.4 \%$ de los casos con dos raíces, y $65.4 \%$ del total de los casos), las RextNAT y la RintNAT formaban un ojal nervioso a la Amm en su trayecto. (Figura 1).

En un caso a izquierda, la RextNAT pasaba entre la Amm y el músculo pterigoideo lateral, y la RintNAT for- 

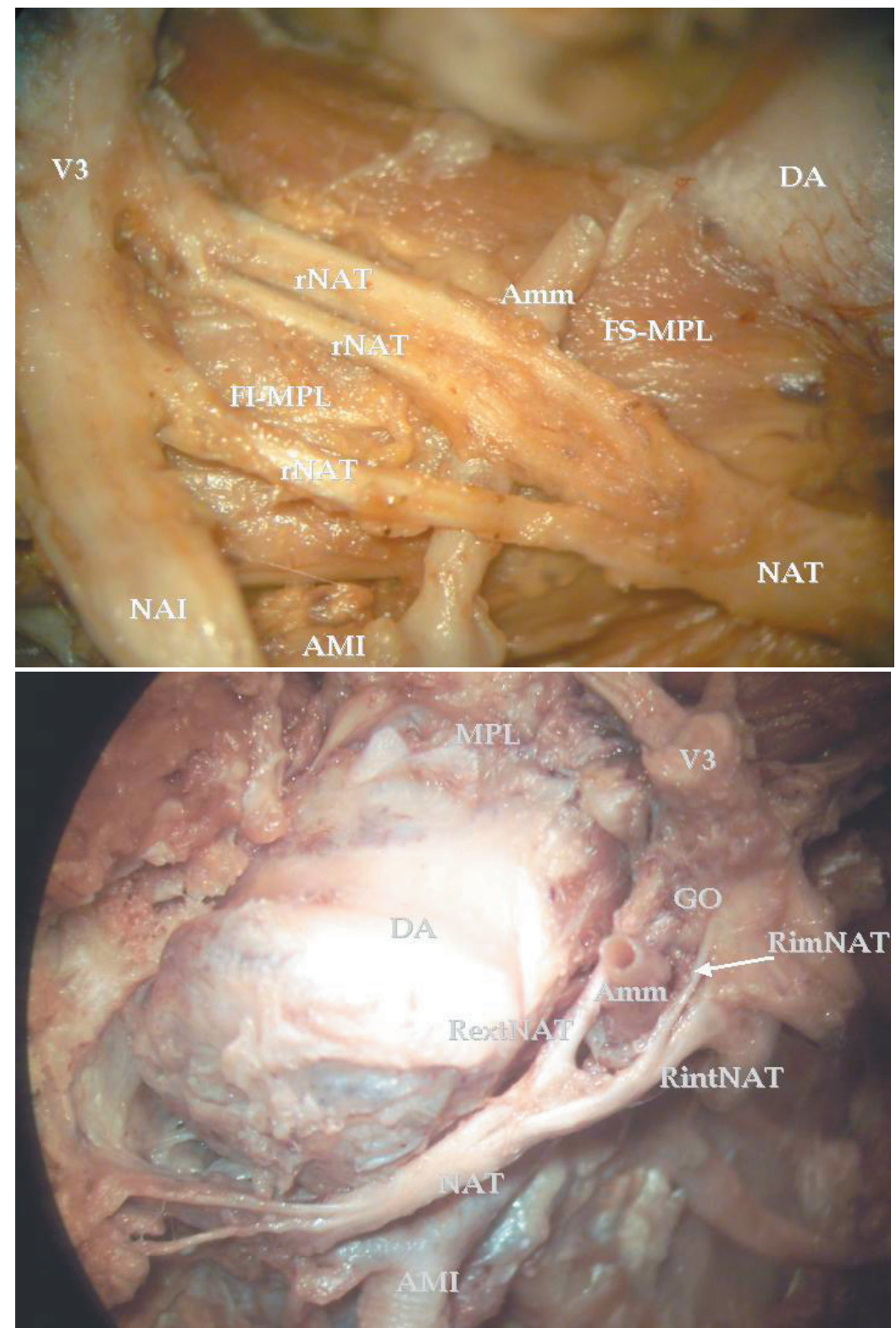

Figura 3. Microdisección de región infratemporal derecha. (Vista medial. Microscopio quirúrgico Olympus. Ampliación x15). Se observa un NAT con tres raices de origen mediales a la AMM. AMI: Arteria maxillaris. Amm: Arteria meningea media. DA: Discus articularis. FI-MPL: Fasciculus inferioris. Mm pterygoidei. FS-MPL: Fasciculus superioris. Mm pterygoidei. NAI: Nervus alveolaris inferior. NAT: Nervus auriculo temporalis. rNAT: Radix NAT. V3: Nervus mandibularis.

Figura 4. Microdisección de región infratemporal izquierda (Vista supero-medial. Microscopio quirúrgico Olympus. Ampliación x15). Se observa el trayecto del NAT en relación con la ATM. AMI: Arteria maxillaris. Amm: Arteria meningea media. DA: Discus articularis. Go: Ganglion oticum. NAI: Nervus alveolaris inferior. NAT: Nervus auriculo temporalis. RextNAT: Radix externa NAT. RimNAT: Radix intermedius NAT. RintNAT: Radix interna NAT. V3: Nervus mandibularis. VMI: Vena maxillaris. maba una ansa por debajo de la arteria maxilar (Arteria maxillaris, AMI) y luego se anastomosaba con la RextNAT. En dos casos a derecha, ambas raíces pasaban medialmente a la Amm.

En cuatro casos (15.4\%), siempre a derecha, se observó una única raíz con origen en el tronco del V3, la cual discurría lateral (dos casos) o medial (dos casos) a la Amm. (Figura 2).

En tres casos (11.5\%), dos a izquierda y uno a derecha, se observaron tres raíces de origen: (1) una RextNAT, superior, con origen en el tronco del V3; (2) una RintNAT, inferior, con origen en el NAI; (3) y una accesoria, intermedia entre las dos antes mencionadas, que se originaba del NAI; en los dos casos a izquierda, las tres raíces formaban un ojal a la Amm, pasando la raíz accesoria y la RintNAT, medialmente a la Amm; en el caso derecho, las tres raíces pasaban medialmente a la Amm. (Figura 3).

En los tres casos la raíz accesoria culminaba fusionándose con la RintNAT.

Debemos jerarquizar que en el caso de existir una raíz con origen en el ganglio ótico, no consideramos a la misma como una raíz de origen del NAT, sino como un ramo anastomótico.

En suma, a izquierda las raíces, siempre fueron 2 ó 3 y siempre formaban un ojal a la Amm en su trayecto; a derecha, las raíces evidenciaron un trayecto más variable, el $65.4 \%$ de estos casos formaban un ojal a la Amm, en $15.38 \%$ todas las raíces eran mediales, en el $7.70 \%$ existía una única raíz de disposición lateral a la Amm y en el 7.70\% existía una única raíz de disposición medial a la Amm. 


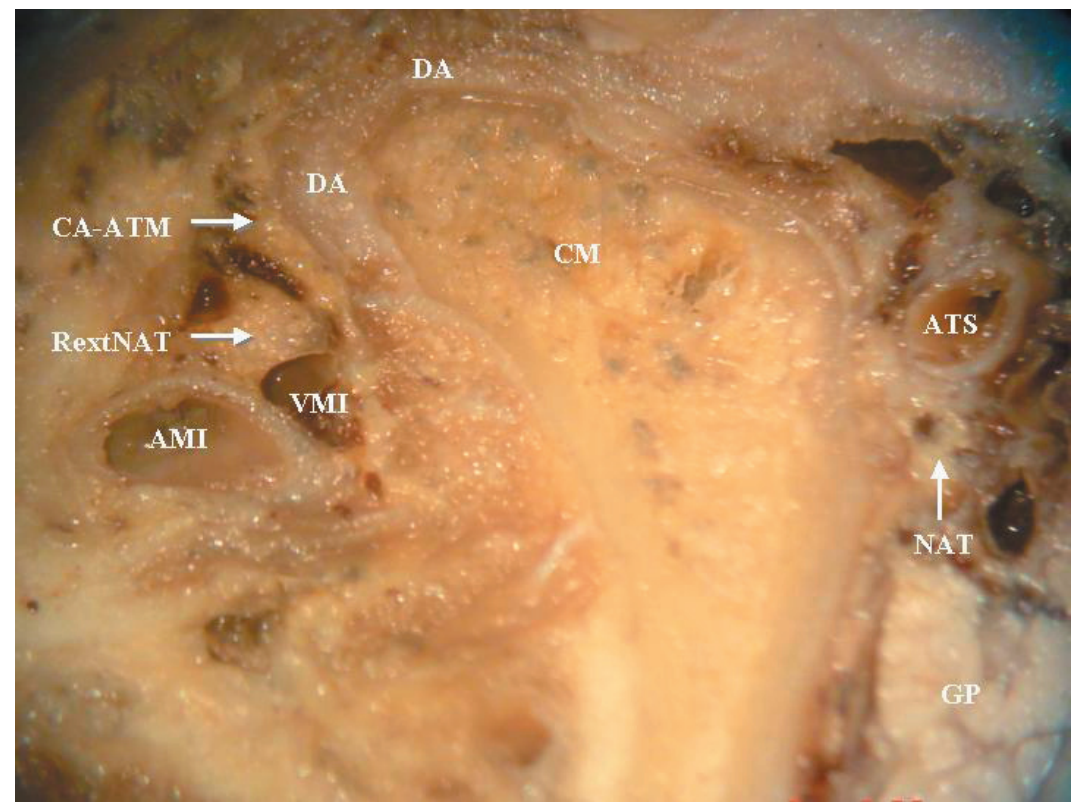

Figura 5. Corte frontal (coronal) de ATM izquierda a nivel del ojal retrocondileo de Juvara (Vista anterior del corte; cadáver humano adulto congelado. Microscopio quirúrgico Olympus. Ampliación x15). Se observa a la raíz lateral del NAT en contacto con la cápsula articular de la ATM. AMI: Arteria maxillaris. ATS: Arteria temporalis superficialis. CA-ATM: cápsula articularis ATM. DA: Discus articularis. NAT: Nervus auriculo temporalis. RextNAT: Radix externa NAT. VMI: Vena maxillaris.

Distancia transversal desde la cara medial de la cápsula articular de la ATM a la cara externa de la RextNAT

Se registro la distancia transversal desde la cara medial de la cápsula articular de la ATM a la cara lateral de la RextNAT en todos los casos.

A derecha, la distancia media fue de $2 \mathrm{~mm}$ (rango: 0-7 $\mathrm{mm}$ ), con una desviación estándar de $1.90 \mathrm{~mm}$. La medida más frecuente encontrada fue de 1,0 mm. En 4 casos ( $21.1 \%$ de los casos en contacto) la RextNAT se encontraba en directo contacto con la cápsula articular de la ATM.

A izquierda, la distancia media fue de $1.25 \mathrm{~mm}$ (rango: 0-3 mm), con una desviación estándar de $0.88 \mathrm{~mm}$. La medida más frecuente encontrada fue de 1,0 mm. En 6 casos (46.1\% de los casos en contacto) la RextNAT se encontraba en directo contacto con la cápsula articular de la ATM.

La distancia media de todos los casos fue de $1,5 \mathrm{~mm}$ (rango: 0-7 mm), con una desviación estándar de 1,76 mm. La medida más frecuente encontrada fue de 1,0 $\mathrm{mm}$. En suma: en 10 casos (31.3\%) la RextNAT se encontraba en directo contacto con la cápsula articular de la ATM. (Figura 4).

\section{Distancia vertical desde el polo medial del disco articular (Discus articularis, DA) de la ATM a la cara superior de la RextNAT}

Se registro la distancia vertical desde el polo medial del disco articular de la ATM a la cara superior de la RextNAT en todos los casos.

A derecha, la distancia media fue de $9 \mathrm{~mm}$ (rango: 5,0$18,0 \mathrm{~mm}$ ), con una desviación estándar de $3,70 \mathrm{~mm}$. La medida más frecuente encontrada fue de $7.0 \mathrm{~mm}$.

A izquierda, la distancia media fue de $11.0 \mathrm{~mm}$ (rango: 3.0-20.0 mm), con una desviación estándar de $4.41 \mathrm{~mm}$. La medida más frecuente encontrada fue de $8.0 \mathrm{~mm}$.

La distancia media de todos los casos fue de $10.5 \mathrm{~mm}$ (rango: 3.5-20.0 mm), con una desviación estándar de 4.06 $\mathrm{mm}$. La medida más frecuente encontrada fue de $11.0 \mathrm{~mm}$.

\section{Relaciones en la región parotídea}

A nivel del ojal retrocondileo de Juvara, las relaciones del NAT fueron constantes, en el $100 \%$ de los casos constituía un pedículo vásculo-nervioso, integrado de arriba abajo por las raíces del NAT, que se fusionaban a este nivel, la vena maxilar (Vena maxillaris, VMI) y la AMI, envueltos en una vaina vascular dependencia de esta última e íntimamente adherida a la cara medial de la cápsula articular de la ATM. (Figura 5).

A nivel de la región parotídea, en la cara posterior de la ATM el NAT formaba en el $100 \%$ de los casos una curva de disposición horizontal en contacto directo con el periostio del borde posterior del cuello del CM; la distancia media vertical entre la cara superior del DA y la cara superior del tronco del NAT a este nivel fue de 12,5 mm (DE: 4,06 mm); a la derecha, la distancia media fue de 11,0 mm (rango: 5$20 \mathrm{~mm}$; DE: 3,70mm); a la izquierda, la distancia fue de 13 $\mathrm{mm}$ (rango: 3,5-22 mm; DE: 4,41 mm).

En 14 casos se registraron las relaciones con la ATS y el tronco del VII; la ATS se encontraba en 8 casos (57\%) posteroinferior al NAT describiendo un trayecto oblicuo de adentro a afuera, de abajo a arriba y de atrás hacia delante, acercándose paulatinamente al NAT con el cual se reúne a nivel del vértice superior de la región parotídea previo a su ingreso en la región temporal como pedículo vásculo-nervioso común; en 6 casos (43\%) la ATS era antero-externa y superior al NAT; la distancia media máxima entre el NAT y la ATS a nivel de la región parotídea es de 2-3 mm. En el 100\% de los casos el tronco del 

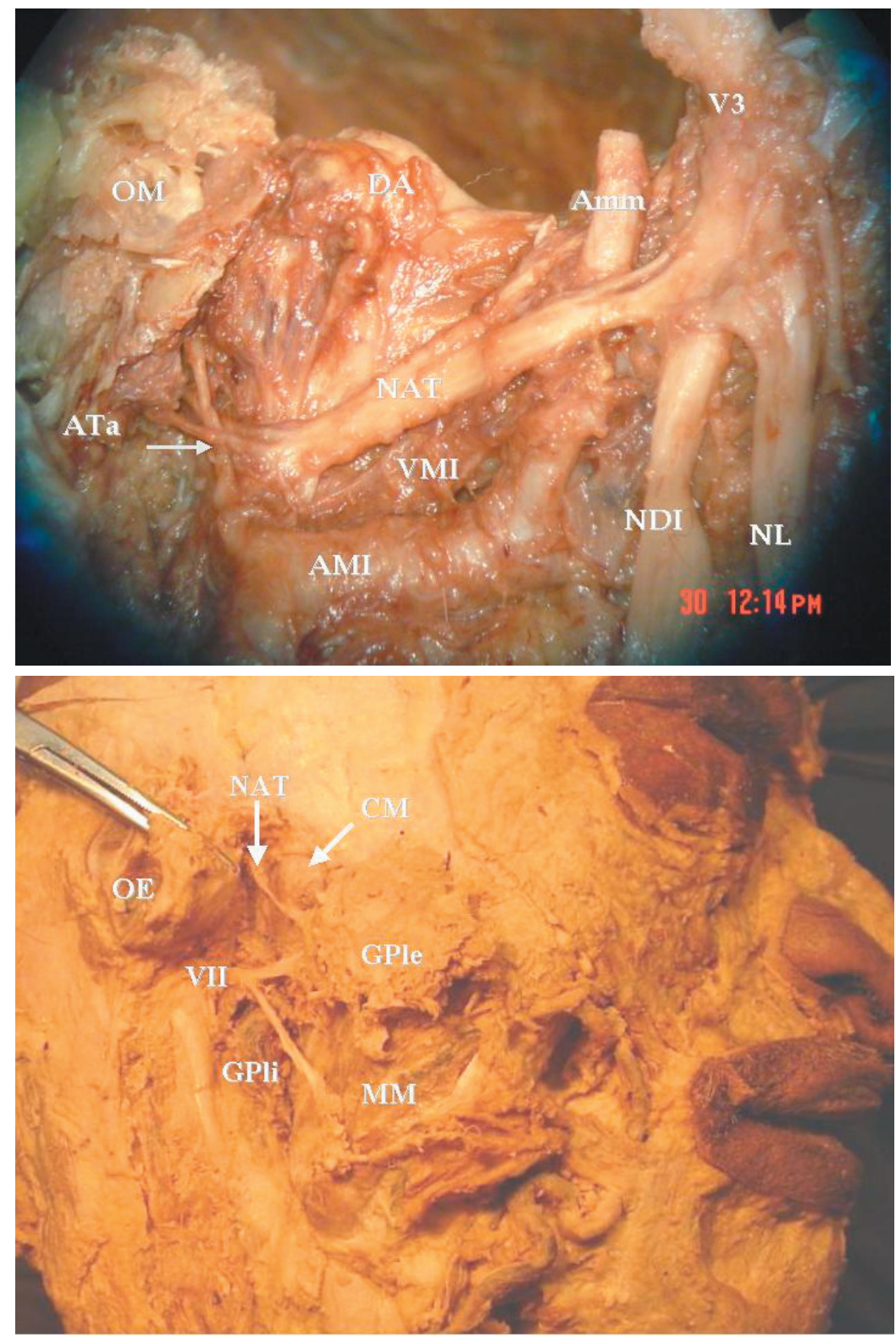

VII y su punto de división terminal se encontraban por debajo, atrás y medial al del NAT.

\section{Ramos colaterales}

En todos los casos se observaron 1 ó 2 ramos con destino a la GP.

Se observaron en 10 casos $(31.25 \%)$ ramos colaterales con destino a la ATM todos originados en el segmento retro-condilar del NAT, y que abordaban a la cápsula articular por su cara postero-externa. El número de ramos colaterales para la ATM promedio fue entre 1-3.

Se observaron en 7 casos (21.9\%) nervus vasorum originados siempre de las raíces del NAT, en particular la RintNAT, con destino a la Amm.

Se observaron en 8 casos $(25 \%)$ ramos óticos, prome-
Figura 6. Microdisección de región infratemporal izquierda (Vista medial. Microscopio quirúrgico Olympus. Ampliación x15). Se observa la pinza nerviosa que forman los ramos oticos del NAT a la Ata. AM: Auris media. AMI: Arteria maxillaris. Amm: Arteria meiíngea media. Ata: Arteria tympánica anterior. DA: Discus articularis. Go: Ganglion oticum. NAI: Nervus alveolaris inferior. NAT: Nervus auriculo temporalis. NL: Nervus lingualis. RextNAT: Radix externa NAT. RintNAT: Radix interna NAT. V3: Nervus mandibularis. VMI: Vena maxillaris.

Figura 7. Disección región parotidea derecha. Se observa al NAT y sus relaciones con el condilo mandibular y el VII. AE: Auris externa. CM: Condylus mandibularis. GPle: Lobus externus Glandula parotis. GPli: Lobus internus Glandula parotis. MM: Musculus masster. NAT: Nervus auriculo temporalis. VII: Nervus facialis. 


\section{Patrón de origen}

En el presente estudio se observó que los patrones de origen son: raíz única (15.4\%), dos raíces $(73.1 \%)$ y tres raíces (11.5\%). A izquierda el origen del NAT estuvo representado por 2 raíces en el $77.8 \%$ de los casos y por 3 raíces en el $22.2 \%$ de los casos; a derecha, el patrón de origen fue más variable, representado por dos raíces en el 70.6\% de los casos, una única raíz en el $23.5 \%$ de los casos y tres raíces en el $5.9 \%$.

En todos estos casos la RextNAT era la de mayor diámetro $(2,0-2,5 \mathrm{~mm})$ y se originaba del tronco del V3 como un ramo colateral posterior, hecho que fue descrito por primera vez por Arnold ${ }^{5}$, y es aceptado por todos los anatomistas clásicos; las restantes raíces eran de menor diámetro y siempre estaban localizadas en un plano medial e inferior con respecto al origen de la RextNAT lo cual se corresponde con lo descrito por los anatomistas clásicos ${ }^{5}$, $7,8,15,17,30,37,38,42,47,50,54,59,61,63,66$. En el 100\% de los casos con 2 o 3 raíces, las raíces mediales e inferiores se originaban del borde posterior del segmento inicial del NAI, y se dirigía de adelante a atrás pasando entre la Amm, lateralmente, y la aponeurosis inter-pterigoidea, medialmente. Esto no coincide con la descripción anatómica clásica de la raíz medial del NAT, la cual es descrita siempre originándose del tronco del V3 al mismo nivel que la raíz lateral.

Estas cifras no están de acuerdo con las descritas por Schmalfuss et $\mathrm{a}^{55}$, quienes describen como patrón de origen constante la existencia de 2 raíces, una lateral y superior, la de mayor diámetro, y otra medial e inferior, de menor diámetro; el presente estudio no está de acuerdo con las cifras registradas por Gulekon, et al. ${ }^{28}$ quienes en un estudio que incluyó 32 NAT observaron los siguientes patrones de origen: raíz única $(50 \%)$, dos raíces $(37.5 \%)$, tres raíces $(9.4 \%)$ y cuatro raíces (3.1\%), estos autores no especifican las relaciones de estas raíces con la Amm; Schmidt et $a^{56}$ en un estudio que incluyo 16 NAT observaron que en todos los casos el origen del NAT estaba formado por una única raíz que se bifurcaba en ojal alrededor de la Amm para luego fusionarse nuevamente en un único tronco nervioso; Vrionis et $\mathrm{al}^{67}$ en un estudio que incluyo 12 NAT observaron que en el 58,3\% de los casos el patrón de origen estaba representado por dos raíces, y en $41.7 \%$ por cuatro raíces. Debemos destacar que estos autores realizaron un abordaje lateral de la fosa infratemporal el cual no permite más que una visión muy limitada del sector medial al MPL, y altera en gran medida la anatomía normal de la región y obliga a realizar maniobras de tracción sobre la ATM que pueden comprometer la anatomía normal del NAT.

\section{Trayecto de las raíces}

En el $100 \%$ de los casos las raíces se dirigían de adelante hacia atrás en la región infra-temporal, en un trayecto de entre 15-16 mm de longitud, entre el MPL y la aponeurosis inter-pterigoidea medialmente, inmediatamente superiores al plano de la VMI y AMI, adquiriendo relaciones variables con la Amm, lo cual no coincide con lo descrito por los anatomistas clásicos $5,7,8,15,17,30,37,38,42,47,50,54,59,61,63,66$ y algunos investigadores actuales ${ }^{55,56,67}$, quienes las describen formando un ojal nervioso constante a la misma. En el $65.4 \%$ de los casos las raíces del NAT formaban un ojal nervioso a la Amm en su trayecto ascendente hacia el foramen spinosum, patrón de relación descrito por primera vez por $\mathrm{Meckel}^{42}$, y asumido como constante por los autores clásicos y actuales previamente citados. Esta relación fue constante a izquierda ( $100 \%$ de los casos); a derecha las raíces evidenciaron un trayecto variable, en el 53\% de los casos las raíces formaban un ojal nervioso a la Amm, en el $23.5 \%$ todas las raíces pasaban mediales a la Amm (16\% de todos los casos), en el $11.7 \%$ existía una única RextNAT a la Amm ( $8 \%$ de todos los casos), este tipo de relación fue descrita por primera vez por Paturet ${ }^{47}$, y en el $11.7 \%$ una única RintNAT a la Amm (8\% de todos los casos). No encontramos en la literatura anatómica descripción de patrones de trayecto mediales a la Amm, representando la presente investigación el primer registro existente sobre este tipo de variaciones en el trayecto de las raíces.

\section{Relaciones en el ojal retrocondíleo de Juvara}

El ojal retrocondíleo de Juvara ${ }^{33}$ está delimitado lateralmente por la cápsula articular de la ATM, medialmente, por el ligamento esfeno-mandibular y superiormente por la base de cráneo en el sector óseo comprendido entre el foramen spinosum y la fisura escamo-esfenoidal. Con una altura de 20-25 mm, el ojal retrocondileo de Juvara comunica la región infra-temporal, que se encuentra por delante con la región parotídea, que se encuentra por detrás. En el presente estudio se evidenció que a través de este foramen osteo-articular y ligamentario pasan de arriba abajo: las raíces del NAT, las cuales se fusionan a este nivel, la VMI y la AMI, envueltos por una vaina neurovascular rica en tejido adiposo y muy adherente a la cara interna de la cápsula articular de la ATM y al MPL y su tendón de inserción discal, lo cual ya fue destacado por Aigrot $^{2} \mathrm{y}$ Hovelacque $^{30}$, y jerarquizado recientemente por Schmidt et al. ${ }^{56}$. Esta vaina neurovascular se continúa con la vaína de tejido conjuntivo denso que envuelve a las raíces del NAT, y el tronco de V3 así como con la duramadre del foramen oval que envuelve el trayecto foraminal de V3, lo cual fue descrito por primera vez por Fawcett ${ }^{24}$.

En el $97 \%$ de los casos las raíces del NAT se fusionaban a nivel del ojal retrocondileo de Juvara; en el 3\% se fusionaban en la región parotídea, lo cual fue descrito por primera vez por Valentin ${ }^{66}$ (plexus gangliosus temporalis internus).

Tomando en consideración la distancia transversal, desde la cara medial de la cápsula articular de la ATM a la cara lateral de la RextNAT, en el presente trabajo, se observó que en el 31.3\% de los casos, la RextNAT, en el 
punto de unión con la RintNAT, se encontraba en contacto directo con la cara medial de la cápsula articular de la ATM, lo cual fue más frecuente a izquierda (46.1\% de los casos) que a derecha (21.1\% de los casos). La distancia media entre la RextNAT y la cara medial de la cápsula articular de la ATM fue de 1,5 mm. A la derecha, la distancia media fue de 2,0 (rango: 0-7 mm), con una mayor variabilidad (DE: $1,90 \mathrm{~mm}$ ). A la izquierda, la distancia media fue de 1,25 mm (rango: 0-3 mm), con una variabilidad menor (DE: 0.88 $\mathrm{mm}$ ). Se destaca la observación de un patrón asimétrico en estas medidas. Estas cifras son superiores a las registradas por Johansson et al. ${ }^{32}$, quienes observaron mediante cortes histológicos que el tronco del NAT se encontraba en directo contacto con la cara medial de la cápsula articular de la ATM en el $16,7 \%$ de los casos, sin especificar las diferencias a izquierda y derecha. Schmidt et al. ${ }^{56}$ investigaron las relaciones del NAT en 16 regiones infratemporales, con la cara medial del CM y del MPL, estos autores observaron que en todos los casos el nervio se encontraba en directo contacto con el CM en el punto de inserción del MPL. Estos autores no especificaron cómo fue considerada la cápsula articular y si el contacto directo implicaba al tronco del nervio o la RextNAT, ya que a nivel del MPL el tronco aun no se ha constituido. Loughner et al. ${ }^{40}$ en un estudio que incluyo 14 cabezas cadavéricas negaron la existencia de relaciones entre el NAT y la cara medial de la cápsula articular de la ATM, que exponen a este nervio a lesión mecánica en los desplazamientos discales. En lo referente a la distancia vertical desde el polo medial del DA a la cara superior de la RextNAT en el ojal retrocondíleo de Juvara, en el presente estudio se observó que la distancia media fue de 10,5 mm (DE: 4,06 $\mathrm{mm}$ ) con un patrón asimétrico significativo: a la derecha, la distancia media fue de 9,0 mm (rango: 5-18 mm), con una variabilidad menor (DE: 3,70 $\mathrm{mm}$ ), a la izquierda, la distancia fue de $11 \mathrm{~mm}$ (rango: 3,5-20 mm), con una variabilidad mayor (DE:4,41 mm). Según Schmidt et al. ${ }^{56}$, la distancia entre el plano de la cara superior del DA y el punto de pasaje medial del NAT es de 3.85-10.27 mm (media: $7.06 \mathrm{~mm}$ ), con un patrón simétrico a izquierda y derecha en la mayoría de los casos, lo cual difiere de lo observado en este trabajo, en el rango más estrecho de sus hallazgos y el patrón simétrico izquierda-derecha. No se encontró en la revisión bibliográfica ningún otro trabajo que estudiara este parámetro en las relaciones de la cara medial de la cápsula articular de la ATM con el NAT.

\section{Relaciones en la región parotídea}

En la región parotídea, en el interior de la GP, el NAT cambia de dirección dirigiéndose hacia afuera, describiendo en el $100 \%$ de los casos una curvatura de concavidad anterior que abraza el cuello del CM en contacto directo con el periostio del mismo, para finalmente llegar a la cara postero-lateral de la cápsula articular de la ATM donde se divide en varias ramas terminales, tal cual lo describen los anatomistas clásicos $5,7,8,15,17,30,37,38,42,47,50,54,59,61,63,66$, la distancia media vertical entre la cara superior del DA y la cara superior del tronco del NAT en el punto en que pasa en contacto con el borde posterior del cuello del CM fue de $12,5 \mathrm{~mm}$ (DE: 4,06 mm) con un patrón asimétrico significativo: a la derecha, la distancia media fue de 11,0 mm (rango: 5$20 \mathrm{~mm}$ ), con una variabilidad menor (DE: 3,70 mm), a la izquierda, la distancia fue de $13 \mathrm{~mm}$ (rango: 3,5-22 $\mathrm{mm}$ ), con una variabilidad mayor (DE:4,41 mm). (Figura 7).

Estos hallazgos están de acuerdo con los registrados por Fernández et al. ${ }^{25}$ quienes observaron que la distancia que separa el plano de la cara superior del CM y el punto de pasaje del NAT en la cara posterior de la cápsula articular de la ATM, es de 10-13 mm. Según Davidson et al. ${ }^{16}$ el NAT se identifica en forma constante, emergiendo de la cara postero-medial del cuello del CM a una distancia aproximada de $12-15 \mathrm{~mm}$ por debajo del plano de la cara superior del CM.

Las relaciones con la ATS son variables, en el 57\% la ATS se encontraba postero-inferior al NAT describiendo un trayecto oblicuo de adentro a afuera, de abajo a arriba y de atrás hacia delante, acercándose paulatinamente al NAT con el cual se reúne a nivel del vértice superior de la región parotídea; en el 43\% la ATS se encontraba por delante y arriba del NAT; la distancia media máxima entre el NAT y la ATS a nivel de la región parotídea es de 2-3 mm. Estos hallazgos no se corresponden con la descripción clásica que describe al NAT pasando en forma constante en su trayecto de medial a lateral, primero medial, luego postero-inferior, y finalmente postero-externo a la $\operatorname{ATS}^{30}$. El presente estudio es el primero en observar y constatar las variaciones en las relaciones del NAT y la ATS en la región parotídea.

En el $100 \%$ de los casos el tronco del VII y su punto de división terminal se encontraban por debajo, atrás y adentro del NAT.

\section{Ramos colaterales}

En todos los casos se observaron 1 ó 2 ramos con destino a la GP. Se observaron en el $31.25 \%$ de los casos ramos colaterales con destino a la ATM todos originados en el segmento retro-condilar del NAT, y que abordan a la cápsula articular por su cara postero-externa, descritos ya por Valentin ${ }^{66}$. El número de ramos colaterales para la ATM promedio fue entre 1-3. Se observaron en el $21.9 \%$ de los nervus vasorum originados siempre de las raíces del NAT, en particular la RintNAT, con destino a la AMM, estos hallazgos están de acuerdo con las primeras descripciones de los mismo por parte de Valentin ${ }^{66}$. Se observaron en el $25 \%$ de los casos ramos óticos, promedio 1 a 3 , que acompañaban a la Ata, medialmente, y, luego de formar una pinza nerviosa a la misma, ingresaban por la fisura tímpano-petro-escamosa de Glasser, los mismos se distribuían 
en la cara interna de la membrana timpánica, ligamento anterior del hueso martillo, ramas de la arteria timpánica anterior y en el conducto auditivo externo. En un 1 caso (3.1\%), a izquierda, se observó un ramo colateral que abordaba el tercio posterior de la cara medial del MPL.

En suma: en el segmento infratemporal se originan los ramos colaterales vasculares con destino a la Amm, el MPL y las anastomosis; en el segmento parotídeo se originan los ramos colaterales articulares, óticos y parotídeos.

En el presente estudio no encontramos a nivel de la región parotídea un plexo nervioso conformado por las ramas colaterales parotídeas del NAT y descrito por Valen$\operatorname{tin}^{66}$ (plexus gangliosus temporalis internus) y donde Cruveilhier ${ }^{15}$ describió la existencia de ganglios nerviosos.

Los hallazgos con respecto a los ramos óticos no están de acuerdo con las descripciones de Schmalfuss I et $\mathrm{al}^{55}$, los cuales describen un único nervio denominado nervio auricular anterior, nervio superior del conducto auditivo externo (ramus meatus auditorii superior) ${ }^{66}$. Rauber en 1865 (citado por Hovelacque ${ }^{30}$ ) describió la existencia de un ramo colateral que se dirige hacia atrás y arriba atraviesa la pared anterior del conducto auditivo externo, en la unión de su parte ósea y su parte cartilaginosa y se distribuye en la piel del mismo y en la membrana del tímpano, lo cual fue evidenciado en el presente estudio; descrito ya por Valentin ${ }^{66}$, el cual lo denominó nervio inferior del conducto auditivo externo (ramus meatus auditorii inferior), se divide en dos ramos, superior e inferior, antes de distribuirse en el pabellón auditivo y conducto auditivo externo; el ramo inferior da origen al plexo de la ATS, el ramo superior se anastomosa con el VII; el ramo temporal superficial, que es origen de múltiples ramos para la cara lateral de la cápsula articular de la $\mathrm{ATM}^{55}$, un nervus vasorum para la ATS; y finalmente, el nervio auricular anterior (ramus auricularis anterior $)^{66}$, se distribuye en el plexo en torno a la ATS, donde se anastomosa con ramas del VII, para luego dividirse en dos ramos terminales anterior y posterior, respecto a la arteria, para la piel de la región temporal.

\section{Anastomosis}

Con respecto a las anastomosis los datos registrados fueron los siguientes:

Se observaron anastomosis con el ganglio ótico (Ganglion oticum, Go) en tres (3) casos, un (1) caso a izquierda, y dos (2) a derecha. El Go es un ganglio nervioso parasimpático anexo al V3 localizado en la cara interna del tronco terminal posterior inmediatamente por debajo del foramen oval. En él encontramos a la $2^{\mathrm{a}}$ neurona para la inervación para-simpática de las glándulas salivales. Sus ramos aferentes son: el nervio petroso menor superficial, con origen en el VII que, transporta las fibras parasimpáticas pre-gangliónicas para la glándulas sub-lingual y sub-maxilar; el nervio petroso menor profundo con origen en el nervio glosofaríngeo (IX par cra- neal) el cual tiene su nacimiento a nivel de la pared interna del oído medio por intermedio del nervio de Jacobson, transporta las fibras para-simpáticas pre-ganglionicas para la GP; y la rama simpática con origen en el plexo simpático peri-vascular de la Amm.

En un caso se observó una anastomosis entre la RintNAT y el NAI, descrito previamente en la literatura ${ }^{30}$.

Se observaron anastomosis con el sistema simpático $0^{5,30,37,47,50,54,59,61,63,66}$, en el curso de su trayecto en la fosa infratemporal importantes anastomosis simpáticas por parte de los plexos peri arteriales de la AMI y la Amm constituidas por fibras nerviosas vegetativas simpáticas con origen en el ganglio cervical superior.

\section{Rol neurovegetativo del NAT}

Con relación al territorio vegetativo, el NAT es la división del nervio mandibular que recibe mayor proporción (50-60\%) de fibras parasimpáticas post-ganglionares originadas en el Go ${ }^{57}$.

\section{Inervación vegetativa de la ATM}

Estudios recientes han demostrado la presencia de terminales nerviosas vegetativas en la ATM de la oveja y la rata ${ }^{12,65}$, a nivel de la cápsula articular, membrana sinovial y periferia del DA, en los frenos discales. Las fibras adrenérgicas de la cápsula articular están asociadas a las arterias de la misma. Estas terminales nerviosas corresponden a fibras nerviosas vegetativas eferentes ${ }^{12}$ y se cree que están involucradas en el control vasomotor de los vasos sanguíneos de la ATM y en la regulación de la secreción del líquido sinovial. Varios ganglios vegetativos dan origen a fibras autónomas postganglionares con destino a la ATM, la contribución porcentual en fibras de cada uno de ellos es la siguiente: ganglio cervical superior (61-71\%), ganglio estrellado (12-26\%) y Go (10-20\%), lo cual indica una marcada preponderancia del sistema nervioso autónomo simpático ${ }^{12}$.

\section{Inervación de la GP}

La GP recibe inervación vegetativa simpática y parasimpática. Los efectores comunes de ambos sistemas son las células acinares secretoras, las células de los conductillos intra e interlobulillares, las células mioepiteliales y el músculo liso del conducto de Stenon.

Pertenecen a la división parasimpática craneal anexa al nervio glosofaríngeo. Representan la vía eferente del arco reflejo salival parasimpático.

El origen de la fibra pre-gangliónica se encuentra en el bulbo raquídeo a nivel del núcleo salival inferior de Kohnstamm $^{35}$ topografíado en el piso del IV ventrículo a nivel del ala gris. Algunos autores niegan la existencia del núcleo salival inferior, debido a que sucesivas investigaciones han demostrado que las neuronas pre-ganglionares forman una población continua en el interior de la sustancia reticular 
bulbo-pontina (nucleus reticularis parvocellularis) ${ }^{4}$, que los autores anglosajones denominan núcleo salival, el cual posee dos componentes, inferior y superior, anexos al IX pc y al VII pc, respectivamente.

Las fibras pre-gangliónicas acompañan a las fibras sensitivas y motoras del nervio glosofaríngeo en su trayecto intracraneal, integrándose luego al nervio timpánico de Jacobson, el cual ingresa en la caja del tímpano, a través de su pared inferior, y da origen a los nervios petrosos profundos; las fibras pre-gangliónicas se integran al nervio petroso profundo menor el cual debe ser considerado como el ramo terminal de este nervio; el trayecto de este nervio es descrito en forma diferente por distintas autoridades anatómicas; según Paturet ${ }^{47}$ y Hovelacque ${ }^{30}$ abandona la caja del tímpano a través de la fisura esfeno-petrosa (fisura petro-timpánica) o el orificio innominado de Arnold (cannaliculus innomina$t u s$ ), este canalículo se encuentra en la delgada lamina ósea que separa al conducto carotídeo del conducto del músculo tensor del tímpano; según Testut-Latarjet ${ }^{63}$ abandona la caja del tímpano por su pared superior a través de los hiatos de Falopio, se une inmediatamente al nervio petroso superficial menor del VII en la cara anterior del peñasco y finalmente abandona la cavidad craneal a través del foramen oval, para anastomosarse con el Go. Los nervios petrosos menores profundo y superficial constituyen la principal aferencia parasimpática (raíz motora o larga) del ganglio ótico. Los autores anglosajones, al igual que los alemanes, no describen al nervio petroso profundo menor; en efecto, estos autores informan de la existencia de fibras anastomóticas entre el nervio de Jacobson y el nervio petroso superficial menor, el cual lo consideran como el portador de las fibras parasimpáticas pre-gangliónicas secreto motoras.

El Go (descubierto por Arnold ${ }^{5}$ en 1826) es el ganglio parasimpático anexo al V3 y responsable del origen de las fibras parasimpáticas post-gangliónicas destinadas al territorio de distribución del mismo, y que acompañan a sus ramos colaterales y terminales.

Según Hovelacque ${ }^{30}$ existen 2 ó 3 ramos anastomóticos eferentes entre el Go y el NAT. Khosravani N et al ${ }^{34}$ observaron que luego de la sección del Go y/o del NAT persistía el $12 \%$ de la actividad refleja parasimpática de la GP, y sugirieron la hipótesis de que las fibras post-gangliónicas parasimpáticas accesorias son aportadas por el VII en su trayecto a través de la GP; no existen aún estudios anatómicos que demuestren la existencia de fibras originadas en el VII con destino a la GP.

Las fibras post-gangliónicas parasimpáticas acompañan con frecuencia a los conductos y se ramifican con libertad alrededor de los acinos.

Estas fibras son de carácter amielínico.

El parasimpático y el simpático tienen un efecto sinérgico sobre la secreción salival, ambos estimulan la misma en forma directa sobre las células epiteliales y estimulan la contracción de los mioepiteliocitos. El antagonismo parasimpático-simpático solo se observa aquí a nivel de la circulación arterial glandular ${ }^{23}$.

Garret $^{26}$ resumió los efectos sobre la GP del siguiente modo: el parasimpático tiene una actividad regulatoria prevalente, la cual se puede dar aisladamente, evoca la mayor parte de la secreción salival, estimula la contracción de las células mioepiteliales y la vasodilatación arterial; el simpático tiene una actividad regulatoria intermitente, sinérgica, modula la composición salival, estimula la contracción de las células mioepiteliales sólo cuando existe una estimulación repetida de las neuronas simpáticas y vasoconstricción arterial.

El estímulo parasimpático produce un flujo salival copioso y acuoso, y que persiste mientras que se aplique el estímulo. El estímulo simpático produce una saliva escasa, rica en solutos orgánicos y ante una estimulación mantenida cesa completamente, este descenso en la respuesta secretora ante el estimulo simpático mantenido se debe al predominio del aumento de la respuesta vasoconstrictora con disminución del flujo sanguíneo.

Las células ductales pueden modificar las características de la saliva, y esto depende de su inervación autónoma.

Se ha sugerido la presencia de fibras vegetativas, parasimpáticas y simpáticas, aferentes que transportan la sensibilidad visceral (interoceptiva) de la GP.

\section{Rol vasomotor}

El presente estudio evidenció la existencia de nervus vasorum originados en el NAT con destino a la Amm.

Walters et $\mathrm{al}^{68}$ demostraron que las neuronas parasimpáticas del Go inervan a la Amm.

Posteriormente se ha demostrado que existen fibras postganglionares parasimpáticas originadas del Go de carácter recurrentes que ingresan nuevamente por el foramen oval y se anastomosan con el plexo nervioso lateroselar ${ }^{53}$, así como fibras post-ganglionares parasimpáticas que tienen un trayecto recurrente en el interior del nervio petroso superficial menor y se anastomosan con el plexo carotídeo interno intracraneal ${ }^{60}$.

Nakai et $\mathrm{al}^{45}$ demostraron que el nervio petroso superficial mayor transporta fibras parasimpáticas pre-ganglionares de carácter vasodilatador, originadas en una población específica de neuronas del núcleo salival superior al cual denominaron centro parasimpático vasodilatador del VII.

El presente estudio plantea la interrogante sobre el posible papel del NAT en el transporta de fibras postganglionares parasimpáticas destinadas a la Amm: ¿cuál es el origen real de las fibras pre-ganglionares parasimpáticas vasodilatadoras con destino al Go, qué nervio craneal las transporta, el glosofaríngeo en su nervio petroso profundo menor, o el VII en su nervio petroso superficial menor? 
Ellrich $\mathrm{J}$ et $\mathrm{al}^{21}$ demostraron en la rata que el nervio trigémino que las fibras aferentes sensitivas somáticas con origen en el territorio cutáneo y las fibras viscerales aferentes con origen en las menínges y sus arterias, convergen en los mismos somas neuronales del sub-núcleo espinal del núcleo sensitivo del trigémino a nivel del bulbo raquídeo y del asta dorsal de la médula espinal de los segmentos $\mathrm{C} 1$ y $\mathrm{C} 2$. ¿El NAT transporta fibras aferentes viscerales de origen vascular meníngeo y vascular extracraneal que culminen en los mismos somas neuronales que reciben la información sensitiva de la ATM y de su territorio cutáneo?

Inervación de los anexos de la piel, red arterial subcutánea, de las regiones temporal, pterional y pabellón auricular

El NAT transporta fibras simpáticas post-ganglionares con destino a los anexos y micro circulación arterial de la piel de las regiónes temporal, pterional y el pabellón auricular. Según la descripción clásica estas fibras son aportadas por anastomosis del NAT con los plexos vegetativos periarteriales de las AMI y ATS a nivel de la región parotídea.

Se ha demostrado la presencia de fibras postganglionares simpáticas en el nervio maxilar (Nervus maxillaris, V2), segunda rama terminal del nervio trigémino, las cuales llegan al nervio trigémino por anastomosis del plexo carotídeo interno con V2 y el ganglio de Gasser a nivel de la pared lateral del espacio lateroselar ${ }^{41}$, lo cual plantea la interrogante si existen anastomosis a este nivel con V3, o con el Go a través del foramen oval.

Investigaciones recientes han demostrado la doble inervación vegetativa, simpática y parasimpática, de la microcirculación de la piel de la cara en primates ${ }^{51}$.

\section{Rol del NAT en el SF}

El SF se caracteriza por eritema cutáneo, sudoración intensa, acompañada de rubor y calor, y en algunos casos dolor, en el territorio cutáneo inervado por el NAT, ante todos estímulo que desencadene el arco reflejo vegetativo de la salivación: estímulos olfatorios, gustativos, la masticación, el pensamiento de un alimento, la ansiedad. En niños puede expresarse solo por el eritema y el calor $^{36,39,43,48}$.

Este síndrome es una complicación frecuente de la parotidectomía ${ }^{49,53}$, parcial o total, en pacientes portadores de tumores parotídeos. Se ha descrito también luego del drenaje de abscesos parotídeos, parotiditis, herpes zoster, traumatismos obstétricos consecuencia del uso del fórceps en niños, traumatismos maxilo-faciales con fractura del cuello o $\mathrm{CM}^{49,62}$, abordajes quirúrgicos pre-auricular de la ATM, en la artroscopia de la ATM. En todos estos casos el inicio de los síntomas tiene una latencia de 5 semanas.

Ha sido descrito también asociado con enfermedades del sistema nervioso central como la siringomielia, ence- falitis, epilepsia y como complicación de los abordajes quirúrgicos del ángulo ponto-cerebeloso ${ }^{27}$. Existen descripciones del mismos en pacientes con diabetes mellitus y sin antecedentes quirúrgicos.

En el caso de los pacientes con antecedentes de parotidectomía, parcial o total, hasta el 30\% reporta síntomas característicos del síndrome, pero sólo el 5-10\% son de significación clínica, pero cuando se realiza la prueba de tinción iodada se demuestra que el $80-90 \%$ presenta el síndrome en su forma sub-clínica (asintomática).

El primer reporte de la existencia de intensa sudoración en el territorio cutáneo del NAT como consecuencia de estímulos gustativos se debe a Kastremsky en 1740. Se asocia la primera descripción a Duphenix en 1757, pero este autor en realidad describe una fístula salival parotídea consecuencia de una lesión del conducto de Stenon ${ }^{18}$.

Barthez (1806) y Dupuy (1816) describen y reproducen los síntomas del síndrome. Baillarger (1853) describe dos nuevos casos. Brown Sequard (1849) y Henle (1855) sufrieron en forma personal los síntomas de este síndrome ${ }^{18}$. Bergounhioux (1859) fue el primero en plantear que el acto de la masticación podía desencadenar el estímulo para la salivación y como consecuencia el síndrome ${ }^{18}$.

En 1898 cuando Langley introduce en la literatura científica el término sistema nervioso autónomo, Paul Raymond sugiere la hipótesis de que el sistema autónomo simpático es el responsable del síndrome. Entre 19011905 Langley desarrolla el concepto de sistema autónomo parasimpático, el cual permitiría consolidar posteriormente la etiopatogenia del síndrome ${ }^{18}$.

Lucja Frey en $1923^{10,18}$ fue la primera en definir a esta entidad como una neuropatía vegetativa consecuencia del compromiso mecánico tanto de las fibras vegetativas postganglionares parasimpáticas y simpáticas del NAT. En su trabajo propone un dibujo del arco reflejo salival que involucra una vía aferente: el NL, cuerda del tímpano, VII, ganglio geniculado, y una vía eferente: el núcleo salival inferior, nervio glosofaríngeo, nervio petroso profundo menor, V3 y Go, e identifica al NAT como el eslabón perdido que permite explicar la sintomatología consecutiva a lesión traumática del mismo. La hipótesis de Frey fue posibilitada por el desarrollo previo del concepto de sistema nervioso autónomo y sus distintas divisiones por Langley entre 1898 y 1905 , así como de los arcos reflejos salivales.

La hipótesis actual con mayor aceptación para explicar el SF es la regeneración anormal de la fibras parasimpáticas post-ganglionares del NAT tras la lesión del mismo ${ }^{18,48,53}$. Las mismas se regeneran en dirección inadecuada y se unen a las terminaciones simpáticas cutáneas. Ante todo estímulo del arco reflejo salival, en vez de una respuesta natural de secreción salivar de origen parotídeo, se desencadena, por intermedio de las terminales simpáticas de la piel, vasodilatación de la circulación arterial subcutánea y 
sudoración localizada.

Otra hipótesis se basa en la teoría de la desnervación autonómica desarrollada por Cannon, planteada al SF por Freedberg y jerarquizada por Emmelin ${ }^{22}$. Si se secciona un grupo de fibras secretomotoras de la GP, simpáticas y parasimpáticas, se desarrolla un fenómeno de hipersensibilidad de las terminales sinápticas de la glándula a la nor-adrenalina y acetilcolina. En una primera etapa que dura 2 días se produce una secreción salival aumentada por la liberación de los neurotransmisores de las terminales nerviosas afectadas, que puede ser de la intensidad de una secreción salival paralítica; la segunda etapa, se caracteriza por el desarrollo de una hipersensibilidad máxima de las terminales del NAT, en todos sus territorios vegetativos, en las posteriores 3 semanas a la sección de las fibras postganglionares y que puede persistir durante años o hasta que se produzca la regeneración.

Se ha descrito el SF consecutivo a la lesión del nervio auricular mayor y de la cadena simpática cervical.

El presente estudio aporta las bases anatómicas que permiten comprender el compromiso del NAT en el curso de las distintas etiologías responsables del SF, así como la conservación de este nervio en los abordajes quirúrgicos de la ATM, la GP y la cara exo-craneana de la base de cráneo. En un estudio previo ${ }^{17}$ describimos las bases anatómicas de una neuropatía periférica del NAT en su segmento medial a la cápsula articular como consecuencia de los desplazamientos antero-mediales del DA de la ATM, que traen como consecuencia síntomas como otalgia y tinnitus. Debemos destacar que no existe ningún estudio que haya realizado la prueba de tinción iodada a los pacientes con desplazamientos antero-mediales del disco articular con compromiso del NAT en su segmento medial a la cápsula articular, por lo cual no existen datos de la prevalencia del SF sub-clínico en estos pacientes; sin embargo, ninguno de los mismos desarrolló la clínica clásica del SF.

Esto nos permite desarrollar la siguiente hipótesis: la etiología del SF que involucra al NAT, se debe al compromiso lesional del mismo en su trayecto medial infratemporal y/o retrocondilar parotídeo, lo cual explica la alta prevalencia de este síndrome en los accesos laterales y posterior de la ATM, así como de la GP. Debido a las estrechas relaciones del NAT con la cápsula articular y DA de la ATM, cualquier grado de movilización del cuello del CM o de la ATM en el curso de la intervención quirúrgica, o la disección del lóbulo externo de la GP, puede comprometer a las fibras vegetativas del mismo. Se ha demostrado que las fibras post-ganglionares tienden a tener una disposición concéntrica superficial en el tronco de los nervios periféricos, por lo cual son las primeras expuestas a la compresión o lesión mecánica.

En un acceso lateral de la región parotídea el tronco del NAT se encuentra a nivel de un área respecto a la cara pos- terior del cuello y CM que mide 2-3 mm en sentido anteroposterior y que comienza a $3,5 \mathrm{~mm}$ por debajo del plano de la inter-línea articular y se extiende hasta $22 \mathrm{~mm}$ por debajo de la misma en sentido vertical.

El presente estudio es el primero a nivel de la literatura médica, que relaciona una ciencia básica, la Anatomía, con el SF, evidenciando las bases anatómicas que nos permitan explicar los hechos clínicos observados empíricamente desde las observaciones de Katremsky.

El presente estudio es el primero en registrar y jerarquizar los siguientes hechos en lo referente a la anatomía del NAT: (1) en todos los casos, la RintNAT se originan del NDI; (2) las relaciones de las raíces de origen con la AMM son variables, conformando un ojal nervioso a la misma en el 65,4\% de los casos; (3) la existencia de nervus vasorum con origen en el NAT destinados a la AMM; (4) las estrechas relaciones de las RextNAT y del tronco del NAT con la cara medial de la cápsula articular de la ATM y su disco articular, con un significativo patrón asimétrico izquierda-derecha, con la cual pueden estar en contacto directo; (5) las estrechas relaciones del NAT con la cara posterior del cuello del CM, con cuyo periostio está en directo contacto; (6) la existencia de dos tipos de variantes en las relaciones del NAT con la ATS; y (7) la presencia de pinzas nerviosas formadas por los ramos óticos a la Ata.

\section{Agradecimientos}

A la Dra. María Gabriela Croce Noguez por su colaboración en la configuración y corrección del manuscrito así como en la elaboración del material iconográfico.

\section{Bibliografía}

1. Aguirrem, A.M., Loreto Vent, C. : Tumores de la parótida. Tratamiento quirúrgico. Rev Chilena de Cirugía 2004 Feb; 56: 51-56.

2. Aigrot : De la rèsection du nerf auriculo-temporal et de ses effects sur le sécrétion parotidienne. Lyon Chrurgicale 1920; 55: 409-413.

3. Akita, K., Shimokawa, T., Sato, T. : An anatomic study of the positional relationships between the lateral pterygoid muscle and its surrounding nerves. Eur J Anat 2003; 7 (Suppl 1): 5-14.

4. Al-Hadithi, B.A.K., Mitchell, J. : The otic ganglion and its neural connections in the rat. J Anatomy 1987; 154: 113-119.

5.Arnold, F.: Handbuch Der Anatomie Des Menschen. Freiburg Im Bresgau; Ed. Herder'sche Verlagshandwung, 1851; pp. 899-913.

6. Barnet Izquierdo, R., Domínguez Fleites, L.M., Muguercia Hunnigan, A.F., Reimondo Cruz, R.A. : Frecuencia y sintomatología de las Disfunciones Temporomandibulares. Rev Cubana Ortod 1998;13: 7-12. 
7. Berry, M.M., Standring, S.M., Bannister, L.H. : Sistema Nervioso. En: Williams PL et al (ed). Anatomía de Gray. $38^{\text {th }}$ ed. Volumen 2. Madrid; Harcourt-Brace, 1998; pp. 1237-1239.

8. Bichat, X. : Anatomie Descriptive. Tome III. Paris; J. S. Chaude Ed, 1829; pp. 210-219.

9. Breen, M.F., Donaldson, J., Mitchell, J., Templeton, D.: Concerning the Origin of Substance P-containing Nerves in Rat Parotid Glands. J Dent Res 1987 Feb; 66: 583.

10. Burton, M.J., Brochwick-Lewinski, M.: Lucja Frey and the auriculotemporal nerve syndrome. Journal of the Royal Society of Medecine 1991; 84: 619-620.

11. Carr, D.H. : Regulation Of Parotid And Submandibular Salivary Secretion In The Sheep. Q J Exp Physiol 1984; 69: 589-597.

12. Casatti, C.A., Frigo, L., Bauer, J.A. : Origin of sensory and autonomic innervation of the rat temporomandibular joint: A retrograde axonal tracing study with the Fluorescent Dye Fast Blue. J Dent Res 1999 March; 78: 776-783.

13. Clayman, M.A., Clayman, S.M., Seagle M.B.: A review of the surgical and medical management of Frey Syndrome. Ann Plast Surg 2006; 57: 581-584.

14. Corbin, K.B. : Observations on the peripheral distribution of fibers arising in the mesencephalic nucleus of the fifth cranial nerve. J Comp Neurol 1949; 73: 153-177.

15. Cruveilhier, J.: Traité D'Anatomie Descriptive. $5^{\text {th }}$ Ed. Tome III. Paris; Librairie De La Faculté De Médécine Labe, 1877; pp. 549-559.

16. Davidson, J.A., Metzinger, S.E., Tufaro, A.P., Lee Dellon, A.: Clinical implications of the innervation of the temporomandibular joint. Journal of Craniofacial Surgery 2003; 14: $235-239$.

17. Decuadro-Sáenz, G., Pérez Brignani, A., Sorrenti, N., Doassans, I., Castro, G., Soria Vargas, V. R.: Relaciones del nervio auriculo-temporal con la cara medial de la cápsula articular de la Articulación Temporomandibular y su asociación con los síntomas oticos de las Disfunciones Temporomandibulares. Medicina Oral (México) 2006 Julio-Septiembre; 8: 101-109.

18. Dunbar, E.M., Singer, T.W., Singer, K., Knight, H., Lanska, D., Okun, M.S.: Understanding gustatory sweating. What have we learned from Lucja Frey and her predecessors? Clin Auton Res 2002; 12: 179-184.

19. Ekström, J.: Non-adrenergic, non-cholinergic reflex secretion of parotid saliva in rats elicited by mastication and acid applied on the tongue. Exp Physiol 1998; 83: 697-700.

20. Ekström, J., Emmelin N.: The Secretory Innervation Of The Parotid Gland Of The Cat: An Unexpected Component. Q J Exp Physiol Cogn Med Sci 1974; 59: 11-17.

21. Ellrich, J., Andersen, O.K., Messlinger, K., ArendtNielsen, L.: Convergence of meningeal and facial afferents onto trigeminal brainstem neurons: an electrophysiological study in rat and man. Pain 1999; 82: 229-237.

22. Emmelin, N.: Degeneration Secretion From Parotid Glands After Section Of The Auriculotemporal Nerves At
Different Levels. J Physiol 1968; 195: 407-418.

23. Emmelin, N.: Nerve Interactions in Salivary Glands. J Dent Res 1987 Feb; 66: 509-517.

24. Fawcett, E.:The relation of the dura mater surrounding the inferior maxillary nerve to the temporo-maxillary articulation. J Anat Physiol 1893 Jan; 27 (Pt 2); 179-180.

25. Fernández, P.R., de Vasconsellos, H.A., Okeson, J.P., Bastos, R.L., Maia, M.L.: The anatomical relationship between the position of the auriculotemporal nerve and mandibular condyle. Cranio 2003 Jul; 21: 165-171.

26. Garrett, J.R.: The Proper Role of Nerves in Salivary Secretion: A Review. J Dent Res 1987 Fe; 66: 387-397.

27. Goldstein, D.S., Pechnik, S., Moak, J., Eldadah, B.: Painful sweating. Neurology 2004; 63: 1471-1475.

28. Gulekon, N., Anil, A., Poyraz, A., Peker, T., Turgut, H. B., Karakose, M.: Variations in the anatomy of the auriculotemporal nerve. Clin Anat 2005 Jan; 18: 15-31.

29. Holmberg, J.: The Secretory Nerves Of The Parotid Gland Of The Dog. J Physiol 1971; 219: 463-476.

30. Hovelacque, A.: Anatomie des nerfs craniens et rachidiens et du systeme grand sympathique chez l'homme. Paris; Librairie Octave Doin, Gaston Doin et Cie Editeurs, 1927; pp. 141-143.

31. Ito, J., Oyagi, S., Honji, I.: Autonomic innervation in the middle ear and pharynx. Acta Otolaryngol Suppl 1993; 506: 90-93.

32. Johansson, A.S., Isberg, A., Isacsson, G.: Radiographic and histologic study of the topographic relations in the temporomandibular joint region: implications for a nerve entrapment mechanism. J Oral Maxillofac Surg 1990 Sep; 48: 953-961.

33. Juvara E.: Anatomie de la región ptérygo-maxilaire. Thèse de Doctorat. Paris; Faculte de Medecine de Paris; 1895

34. Khosravani, N., Sandberg, M., Ekström, J.: The otic ganglion in rats and its parotid connection: cholinergic patthways, reflex secretion and a secretory role for the facial nerve. Exp Physiology 2006; 91: 239-247.

35. Kohnstamm, O.: Der nucleus salivatorius chordae tympani (nervi intermedii). Anatomischer Anzeiger 1908 März; 21: 362-363.

36. Labarta, N., Olaguibel, J.M., Gómez, B., et al.: Síndrome del nervio auriculotemporal. Diagnóstico diferencial con alergia alimentaria. Alergol Inmunol Clin 2002; 17: 223-226

37. Latarjet, M., Ruiz Liard, A., Pro, E.: Anatomía Humana. $4^{\text {th }}$ ed. Tomo 1. Buenos Aires; Ed. Médica Panamericana, 2004; pp. 345-346.

38. Lazorthes, G.: Sistema Nervioso Periférico: Descripción, sistematización y exploración. Barcelona; Toray-Masson S. A. , 1976; pp. 76.

39. Llanos de la Torre, M., Vázquez Doval, F.J.: Síndrome de Frey o síndrome del nervio auriculotemporal. Ped Rur Ext 2004; 34 (318): 45-46.

40. Loughner, B.A., Gremillion, H.A., Mahan, P.E., Watson, R.E.: The medial capsule of the human temporoman- 
dibular joint; J Oral Maxillofac Surg 1997 Apr; 55: 363-369.

41. Matthews, B., Robinson, P.P.: The course of postganglionic sympathetic fibers distributed with the trigeminal nerve in the cat. J Phsyiol 1980; 303: 391-401.

42. Meckel, G.F.: Manuale di Notomia Generale, Descrittiva e Patologica. $2^{\circ}$ edizione. Volume II. Napoli; Ed. G Testa, 1843. pp. 355-356.

43. Moreno-Arias, G.A., Grimalt, R., Llusa, M., Cadavid, J., Otal, C., Ferrando, J.: Frey's Syndrome. J Pediatr 2001; 138: 246.

44. Nachlas, N.E., Johns, M.E.: Fisiología de las glándulas salivales. Otorrinolaringología. Paparella, MM et al (ed); $3^{\circ}$ Edición; Volumen 1. Madrid; Ed. Medica Panamericana, 1994; pp. 467-485.

45. Nakai, M., Tamaki, K., Ogata, J., Matsui, Y., Maeda, M.: Parasympathetic cerebrovasodilator center of the facial nerve. Circulation Research 1993; 72: 470-475.

46. Nordenfelt, I.: On The Sympathetic Innervation Of The Parotid Gland Of The Cat. Q J Exp Physiol Cogn Med Sci 1965; 50: 62-64

47. Paturet, G.: Traité D'Anatomie Humaine. Tome IV. Paris; Ed. G. Doin, 1964. pp. 759-772.

48. Reche Frutos, M., García Ara, M.C., Boyano, T., Díaz Pena, J.M.: Síndrome auriculotemporal. Allergol et Inmunopathol 2001; 29: 33-34.

49. Ribeiro, A.M., Da Veiga Neto, A.R.: Aspectos Anatomofisiológicos do Nervo Auriculotemporal e suas Implicações nas Clínicas Odontológica e Médica. Ciências Biológicas e da Saúde 2003 Jan; 24: 77-84.

50. Rouvièrè, H., Delmás, A., Delmás, V.:Anatomía Humana: descriptiva, topográfica y funcional. $11^{\text {th }}$ ed. Volumen I. Barcelona; Ed. Masson, 2005; pp.. 291-292.

51. Ruocco, I., Cuello, A.C., Parent, A., Ribeiro-Da-Silva, A.: Skin blood vessels are simultaneosly innervated by sensory, sympathetic, and parasympathetic fibers. J Comp Neurol 2002; 448: 323-336.

52. Ruskell, G.L.: Distribution of otic postganglionic and recurrent mandibular nerve fibers to the cavernous sinus plexus in monkeys. J Anatomy 1993; 182: 187-195.

53. Santos, R.C., de Salles Chagas, J.F., Pinto Becerra, T.F., Baptistella, J.E., Pagani, M.A., Rocha Melo, A.: Frey syndrome prevalence after partial parotidectomy. Rev Bras Otorrinolaringol 2006; 72: 112-115.

54. Sappey, C.: Tratado de Anatomía Humana. $2^{\text {th }}$ Ed. Tomo 3. Madrid; Ed. Carlos Bailly-Bailliere, 1874; pp. 280-281

55. Schmalfuss, I.M., Tart, R.P., Mukherji, S., Mancuso, A.A.: Perineural Tumor Spread Along the Auriculotemporal Nerve. AJNR Am J Neuroradiol 2002 Feb; 23: 303-311.

56. Schmidt, B.L., Pogrel, M.A., Necoechea, M., Kearns, G.: The distribution of the auriculotemporal nerve around the temporomandibular joint. Oral Surg Oral Med Oral Pathol Oral Radiol Endod 1998 Aug; 86: 165-168.

57. Segade, L.A., Suárez Quintanilla, D., Suárez Núñez,
J.M.: The postganglionic parasympathetic fibers originating in the otic ganglion are distributed in several branches of the trigeminal mandibular nerve: an HRP study in the guinea pig. Brain Res 1987 May; 411: 386-390.

58. Sessle, B.J.: The neural basis of temporomandibular joint and masticatory muscle pain. J Orofac Pain 1999; 13: 238-245.

59. Shankland, W.E.: The Trigeminal Nerve. Part IV: The Mandibular Division; Cranio 2001 July; 19: 153-161.

60. Shimizu, T.: Distribution and pathways of the cerebrovascular nerve fibers from the otic ganglion in the rat: anterograde tracing study. J Auton Nerv Syst 1994 Sept; 49: 47-54.

61. Soulié, A.: Nerfs craniens et rachidiens. En: Poirier P, Charpy A (ed). Traité D'Anatomie Humaine. $2^{\text {th }}$ ed. Tome III. Fascicule II. Paris; Masson et Cie Ed, Libraires de l'Academie de Medecine, 1901; pp. 704-706.

62. Sverzut, C.E., Trivellato, A.E., Souza Serra, E.C., Prado Feraz, E., Sverzut, A.T.: Frey's Syndrome After Condylar Fracture: Case Report. Braz Dent J 2004; 15: 159-162.

63. Testut, L., Latarjet, A.: Tratado de Anatomía Humana. $8^{\text {th }}$ ed. Tomo III. Barcelona. Buenos Aires; SALVAT Editores SA, 1942; pp. 120.

64. Torres, T.S., Lucena Neto, B., Silva, G.O., et al.: Anatomía quirúrgica del nervio auriculotemporal en el acceso preauricular. Int J Morphol 2004; 22: 327-330.

65. Uddman, R., Grunditz, T., Kato, J., Sundler, F.: Distribution and origin of nerve fibers in the rat temporomandibular joint capsule. Anat Embryol (Berl) 1998 Apr; 197: 273-282.

66. Valentin, G.: Traité de Neurologie. Paris; JB Bailliere, Librairie de 1'Academie Royale de Medecine, 1843; pp. 369374.

67. Vrionis, F.D., Cano, W.G., Heilman, C.B.: Microsurgical anatomy of the infratemporal fossa as viewed laterally and superiorly. Neurosurgery 1996 Oct; 39: 777-785.

68. Walters, B.B., Gillespie, S.A., Moskowitz, M.A.: Cerebrovascular Projections From The Sphenopalatine And Otic Ganglia To The Middle Cerebral Artery Of The Cat. Stroke 1986; 17: 488-494.

69. Weijnen, J.A.W.M., Surink, S., Verstralen, M.J.M., Moerkerken, A., De Bree, G.J., Bleys, R.L.A.W.: Main Trajectories Of Nerves That Traverse And Surround The Tympanic Cavity In The Rat. J Anatomy 2000; 197: 247-262.

Decuadro-Sáenz, G.; Castro, G.; Sorrenti, N.; Doassans, I.; Deleon, S.; Salle, F.; Saibene, A.; Santamaría, A.; Pérez- Brignani, A.; Soria-Vargas, V.R.: El nervio auriculotemporal. Bases neuroanatómicas del Síndrome de Frey. Neurocirugía 2008; 19: 218-232.

Correspondencia postal: Germán Decuadro Sáenz. Departamento de Anatomía. Facultad de Medicina. Universidad de la República. Gral. Flores 2125, Montevideo, Uruguay. Código Postal: 11800. 